\title{
Three-Dimensional Liver Reconstruction Based on Marching Cube and Revised Laplacian Smoothing
}

\author{
Tan Ke (The Lead Author) \\ Educational Technology Center \\ The Chinese PLA General Hospital \\ Beijing, PR China \\ Tanke123@sina.com
}

\author{
Gao Yuan (CorrespondingAuthor) \\ Educational Technology Center \\ The Chinese PLA General Hospital \\ Beijing, PR China \\ Gaoyuan301@126.com
}

\begin{abstract}
In the three-dimensional liver reconstruction, in order to decrease shrinkage and make up the lost important details in Laplacian smoothing, we make some improvements to revise the algorithm in this paper.The cost function after Laplacian smoothing process is constructed to make up the lost important details. When the value of cost function is minimum, the result is close to original liver volume. From the results, we can see that volume of the revised algorithm is more closely to the original volume compared with Laplacian smoothing algorithm.
\end{abstract}

Keywords: Marching cube algorithm; Revised Laplacian smoothing; 3D reconstruction; Liver Reconstruction

\section{INTRODUCTION}

Primary hepatocellular carcinoma (HCC) and metastatic liver cancer are refractory malignancies. The alternative treatment mainly includes surgical operation, minimally invasive surgery and living donated liver transplantation (LDLT). By reason of the anatomical structure complexity and intrahepatic vascular variability, and patients with HCC often have chronic liver disease, such as chronic hepatitis, cirrhosis, preoperative planning of the liver resection volume, area around the tumor and relationship of intrahepatic vessels should be typically evaluated.

The majority of work performed in radiology is presented as 2-D information, from conventional $\mathrm{x}$-ray images to the most advanced computed tomography (CT), magnetic resonance imaging (MRI) or ingle-photon emission computed tomography (SPECT) studies[1,2]. Advances in radiological techniques have allowed three-dimensional (3D) hepatic reconstruction from multiple 2D slices of CT or MRI images, which can provide the complex anatomy present in slices and volumetric information [3-7]. 2D medical images may require special training, and although radiologists have these skills, they must often communicate their interpretations to the referring physicians, who sometimes have difficulty visualizing the $3 \mathrm{D}$ anatomy. Therefore, $3 \mathrm{D}$ reconstruction is useful to understand tumor localization and to make a resection proposal.

The algorithm of 3D reconstruction mainly includes surface rendering and volume rendering. Marching cube (MC) algorithm creates triangle models of constant density surfaces from 3D medical data[8-10]. It is the most common method in surface rendering. Because of the segmented data is mask volume data, so Laplacian smoothing is used to smooth a polygonal mesh [11,12]. But there are some disadvantages in smoothing operation, for example, it reduces high frequency information in the geometry of the mesh. With excessive smoothing important details may be lost. Therefore, with the aim of decreasing the shrinkage, we make some improvements to Laplacian smoothing in this paper.

\section{MATERIALS AND METHODS}

\section{A. Marching cube algorithm}

Marching squares algorithm we can adapt our approach to the $3 \mathrm{D}$ case: this is the marching cubes algorithm. In a 3D space we enumerate 256 different situations for the marching cubes representation. All these cases can be generalized in 15 families by rotations and symmetries (Figure 1).

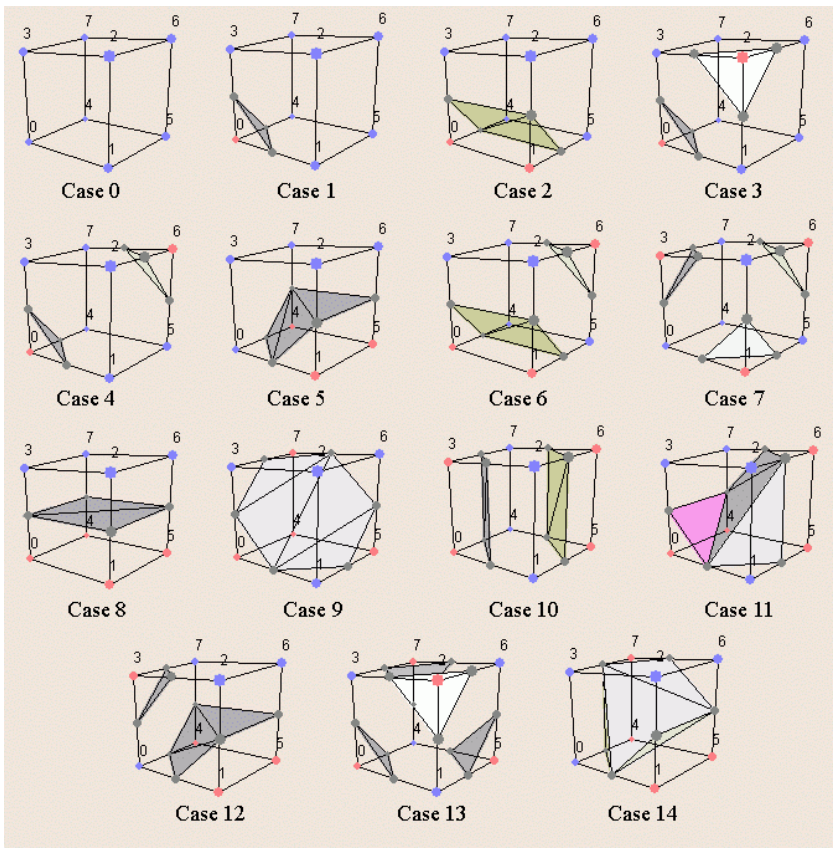

Fig. 1. It represents the 15 families from the marching cubes algorithm

In order to be able to determine each real case, a notation has been adopted. It aims at referring each case by an index created from a binary interpretation of the corner weights. 
In this way, vertexes from 1 to 8 are weighted from 1 to 128 ( $11=1$, v2 = 2, v3 = 4, etc.); for example, the family case 3 example you can see in the picture above, corresponds to the number 5 (v1 and v3 are positive, $1+4=5$ ).
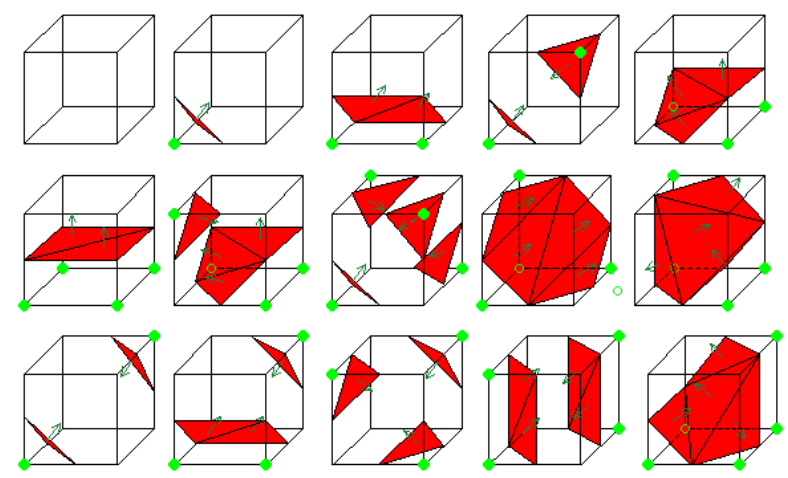

Figure 2 The creation of these complementary cubes allows giving an orientation to the surface.

\section{B. Laplacian Smoothing}

The Laplacian smoothing technique changes the position of nodes without modifying the topology of the mesh. Each internal surface node is moved to a new position given by the average of nodes connected to it by an edge. This works fine for triangular or quadrilateral meshes. For mixed meshes, however, this simple scheme behaves not fully satisfactorily. Let's assume, for example, the mesh depicted on the left consisting of ideal elements only (squares and equilateral triangles). Employing the standard Laplacian smoothing will move the nodes on the interface between the quadrilaterals and triangles towards the triangles and consequently deteriorate the quality of the elements. This effect can be eliminated by introducing weighted Laplacian smoothing with different weights for nodes connected to the smoothed node by an edge shared

by two quadrilaterals ( $\omega=3-\sqrt{3}$ )

by one quadrilateral and one triangle ( $\omega=3-\sqrt{1}$ )

by two triangles $(\omega=1)$

These weights can be determined using a "do not harm" concept, the idea of which is to not move the node shared by elements of ideal shape (Fig. 7).

The Laplacian smoothing can be further extended by weights accounting for the valence (connectivity number) of nodes connected to the smoothed node. It is well known that nodes with a valence larger (smaller) than the ideal one tend to attract (repulse) the smoothed node. Again, the setup of the weights for triangular and quadrilateral meshes is quite easy, because the ideal valence is evident. In a mixed mesh, however, the determination of the ideal valence is not straightforward. The following formulas for the ideal valence $v_{i}$ of an internal node and $v_{b}$ of a boundary node in a mixed mesh proved to be beneficial.

$$
v_{i}=\frac{12(q+t)}{3 q+2 t}, v_{b}=\frac{12(q+t)}{3 q+2 t} \frac{\omega}{360}+1
$$

where $q$ and $t$ stand for number of quadrilaterals and triangles sharing the node and $\omega$ is the angle (in degrees) in the surface tangent plane at the boundary node filled in by the surface.

Note that the repositioning of a node is likely to shift the node out of the surface (unless the surface is planar). Therefore the projection must be employed to satisfy the surface constraint.

\section{Revised Laplacian smoothing algorithm}

Because of the segmented data is mask volume data, so Laplacian smoothing is used to smooth a polygonal mesh (Figure 3). But there are some disadvantages in smoothing operation, the surface may shrink towards the centroid (hrinkage) and the volume will become smaller (Figure 4).

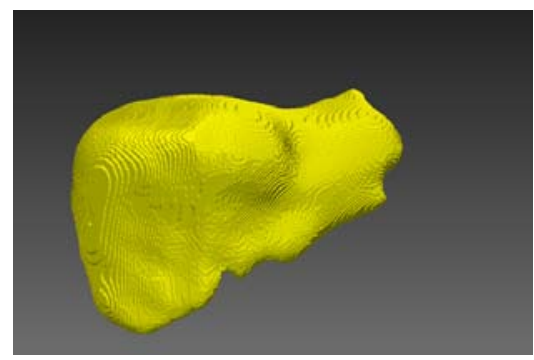

Figure 3 It is a polygonal mesh created by Marching cube algorithm.

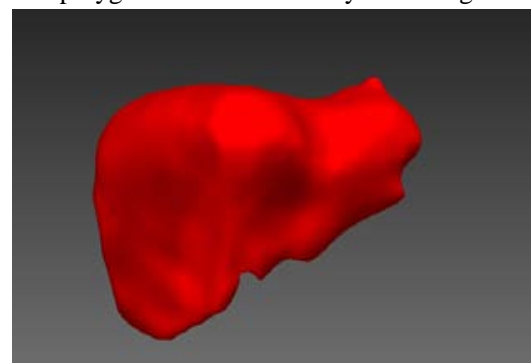

Figure 4 It is a polygonal mesh after Laplacian smoothing process

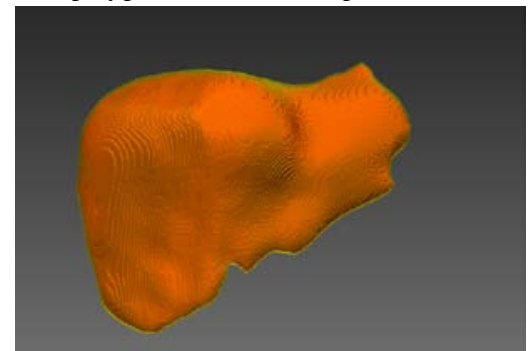

Figure 5 The image is the composition of figure 3 and figure 4 .

Therefore, we improved the Laplacian smoothing algorithm and decreased the shrinkage problem in the smoothing process. We constructed the cost function after smoothing process.

$$
\begin{aligned}
& j(\theta)=\sum_{i=1}^{m}\left(d_{1}+d_{2}+\ldots+d_{n}\right) \\
& d_{n}=\left|V_{\text {Point }}\right| *\left|V_{\text {normal }}\right| \cos (a) \\
& \min j(\theta)
\end{aligned}
$$


$d_{1} \quad \ldots d_{n}$ is the projector distance of the point from the original mesh to all the points in the containing cells along the normal direction.

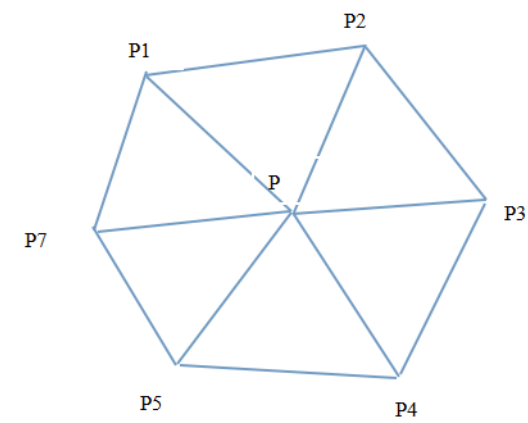

Figure 6

$$
\begin{aligned}
& V_{t}=\sum_{i=0}^{n}\left(V_{p 1-p}+V_{p 2-p}+\ldots+V_{p n-p}\right) \\
& V_{\text {Dir }}=\left(P_{\text {normal }}+V_{t} * a\right)
\end{aligned}
$$

$\mathrm{V}_{\mathrm{p} 1-\mathrm{p}} \ldots \mathrm{V}_{\mathrm{pn}-\mathrm{p}}$ is the projector Vector of the point from the original mesh to all the points in the containing cells. $V_{\text {Dir }}$ is the Direction of movement. Increase parameter $\mathrm{V}_{\mathrm{t}}$ is to prevent the mesh structural changes.

Therefore, when the value of cost function is minimum, the result is close to original liver volume.

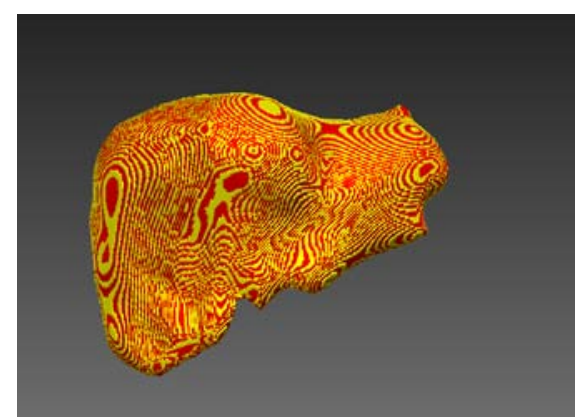

Figure 7The image is the composition of originalmesh and polygonal mesh created by revised Laplacian smoothing.

Table 1 The comparison of volumes from Laplacian smoothing and revised algorithm.

\begin{tabular}{|c|c|c|c|c|c|}
\hline Case & $\begin{array}{c}\text { Original vlume } \\
\text { (mask data ) } \\
\text { (ml) }\end{array}$ & $\begin{array}{c}\text { Volume after } \\
\text { Laplacian } \\
\text { smoothing (ml) }\end{array}$ & $\begin{array}{c}\text { Volume of } \\
\text { revised } \\
\text { algorithm (ml) }\end{array}$ & $\begin{array}{c}\text { L/O(ratio of } \\
\text { laplacian } \\
\text { volume to } \\
\text { original) }\end{array}$ & $\begin{array}{c}\text { R/O(ratio of } \\
\text { revised volume } \\
\text { to original) }\end{array}$ \\
\hline $\mathbf{1}$ & 1573 & 1482 & 1562 & 0.942 & 0.993 \\
\hline $\mathbf{2}$ & 1724 & 1653 & 1734 & 0.959 & 1.006 \\
\hline $\mathbf{3}$ & 1606 & 1509 & 1624 & 0.940 & 1.011 \\
\hline $\mathbf{4}$ & 1733 & 1606 & 1741 & 0.927 & 1.005 \\
\hline $\mathbf{5}$ & 1681 & 1604 & 1675 & 0.954 & 0.996 \\
\hline $\mathbf{6}$ & 1533 & 1484 & 1538 & 0.968 & 1.003 \\
\hline
\end{tabular}

\section{RESULTS}

Figure 5 shows the composition of Figure 3 and Figure 4. Figure 3 is a polygonal mesh created by Marching cube algorithm. Figure 4 is a polygonal mesh after Laplacian smoothing process. From the composition result, we can see that polygonal mesh created by Laplacian smoothing is smaller than original mesh. And the Laplacian polygonal mesh is almost fully hidden by original liver model. From the Figure 6, we can see that original mesh and revised polygonal mesh are somewhat overlapped.

From the results shown in Table 1 , we take case 1 as an example. The original volume is $1573 \mathrm{ml}$, volume after Laplacian smoothing is $1482 \mathrm{ml}$, volume of revised algorithm is $1562 \mathrm{ml}$. The ratio of Laplacian volume to original is 0.942 , and volume of revised algorithm to original is 0.993. Obviously, volume of the revised algorithm is more closely to the original volume compared to Laplacian smoothing algorithm. From the remaining 5 cases, results are the same. The mean \pm SD of the $\mathrm{L} / \mathrm{O}$ is $0.948 \pm 0.015$. The mean $\pm \mathrm{SD}$ of the $\mathrm{R} / \mathrm{O}$ is $1.002 \pm 0.007$. By analyzing the data using independent sample $t$ test, the result shows remarkable difference $(\mathrm{p}<0.001)$.

\section{DISCUSSION}

Advances in radiological techniques have allowed threedimensional (3D) hepatic reconstruction from multiple 2D slices of CT or MRI images, which can provide the complex anatomy present in slices and volumetric information. The algorithm of 3D reconstruction mainly includes surface rendering and volume rendering. Marching cube (MC) algorithm creates triangle models of constant density surfaces from 3D medical data. Because of the segmented data is mask volume data, so Laplacian smoothing is used to smooth a polygonal mesh. But there are some disadvantages in smoothing operation, for example, it reduces high frequency information in the geometry of the mesh. With excessive smoothing important details may be lost. The surface may shrink towards the centroid (Shrinkage) and the volume will become smaller. We constructed the cost function after smoothing process in this paper.

From the results, we can see volume of revised algorithm is more closely to original volume (mask data) compared to Laplacian smoothing. And the mean $\pm \mathrm{SD}$ of the $\mathrm{L} / \mathrm{O}$ is $0.948 \pm 0.015$. The mean $\pm \mathrm{SD}$ of the $\mathrm{R} / \mathrm{O}$ is $1.002 \pm 0.007$. By analyzing the data using independent sample $t$ test, the result shows remarkable difference $(\mathrm{p}<0.001)$. Therefore, the improved Laplacian algorithm can make up the lost important details from Laplacian smoothing.

By means of this, preoperative planning of the liver resection volume, area around the tumor and relationship of intrahepatic vessels as well as virtual surgery of hepatic cancer could be precisely evaluated.

\section{ACKNOWLEDGMENT}

This work is supported by Beijing Natural Science Foundation( 4132080 ). 


\section{REFERENCES}

[1] PHILLIPS W A, KLING T F. MRI and CT evaluation of primary bone and soft-tissue tumors[J]. American Journal of Roentgenology, 1986, 146(4): 749-756.

[2] NG S H, CHANG T-c, KO S F, et al. Nasopharyngeal carcinoma: MRI and CT assessment[J]. Neuroradiology, 1997, 39(10): 741-746.

[3] KATZ M, KONEN E, ROZENMAN J, et al. Spiral CT and 3D image reconstruction of vascular rings and associated tracheobronchial anomalies.[J]. Journal of Computer Assisted Tomography, 1995, 19(4).

[4] PÖTTERA R, CHRISTINE M H, ERIK V, et al. Recommendations from gynaecological (gyn) GEC estro working group (ii): concepts and terms in 3D image-based treatment planning in cervix cancer brachytherapy - 3D dose volume parameters and aspects of 3D imagebased anatomy, radiation physics, radiobiology[J]. Radiotherapy and Oncology, 2006, 78(1): 67-77.

[5] KHAMBAY,BNEBEL, JCBOWMAN, et al. 3D stereophotogrammetric image superimposition onto $3 \mathrm{D}$ CT scan images: the future of orthognathic surgery. a pilot study.[J]. The International Journal of Adult Orthodontics and Orthognathic Surgery, 2002, 17(4): 331-341.

[6] HEINZ K H, RALPH B. Shading 3D-Images from CT using Gray-Level gradients[J]. Medical Imaging, IEEE Transactions, 5(1): 45-47.

[7] HEINZ K H, HANSON A W. Interactive 3D segmentation of MRI and CT volumes using morphological operations.[J]. Journal of Computer Assisted Tomography, 1992, 16(2).

[8] Marcos Vinicius Mussel Cirne, HélioPedrini. Marching cubes technique for volumetric visualization accelerated with graphics processing units.[J].Journal of the Brazilian Computer Society,2013,19(3): 223-233.

[9] LisCustodio,TiagoEtiene,SinesioPesco,ClaudioSilva.Practical considerations on Marching Cubes 33 topological correctness.[J].Computers \& Graphics.2013, 37(7): 840-850.

[10] Gerard L. VignolesLis,Custodio, Marc Donias, ChristianneMulat. Simplified marching cubes: An efficient discretization scheme for simulations of deposition/ablation in complex media.[J]. Computational Materials Science.2011, 50(3): 893-902.

[11] B. Yahiaoui, H. Borouchaki, A. Benali, C. Bennis. Optimization of Dynamic 3D Hex-dominant Mesh Adapted for Basins Simulation Using the Smoothing Laplacian 2D.[C]. ECMOR XIII - 13th European Conference on the Mathematics of Oil Recovery,10 September 2012,242-258.

[12] DimitrisVartziotisa, Joachim Wipperc.A dual element based geometric element transformation method for all-hexahedral mesh smoothing.[J]. Computer Methods in Applied Mechanics and Engineering, 2011, 200(9-12): 1186-1203. 